\section{Note zur Theorie der schlichten Funktionen}

von

W. WIRTINGER (Wien).

In der Abhandlung „Beiträge zur Theorie der schlichten Funktionen" entwickelt Herr Z. W. Birnbaum auf Seite 167 und 168 der Studia Mathematica 1 (1929) eine Ungleichung für im Äußern des Einheitskreises reguläre und schlichte Funktionen, welche dieses Äußere auf das Äußere eines konvexen Bereiches abbilden. Er bedient sich dabei der Abbildung des Einheitskreises auf das Äussere eines konvexen Polygons, welche durch die Funktion

$$
f(z)=\int_{1}^{z} \Pi\left(z-z_{k}\right)^{t_{k}} \frac{d z}{z^{2}}
$$

gegeben ist, wo $0<\alpha_{k}<1, \sum \alpha_{k}-2$ ist, und die $z_{k}$ auf dem Einheitskreis liegen. Hiezu muß aber noch die weitere, dort nicht. angeführte Bedingung treten:

$$
\sum \alpha_{k} z_{k}=0
$$

weil sonst in der Umgebung der unendlich fernen Stelle ein Glied mit $l(z)$ auftreten würde.

Es ist bemerkenswert, daß auf Grund dieser Bedingung die Ungleichung des Herrn BirnBaum

$$
\left|\operatorname{Arg} f^{\prime}(z)\right| \leqslant 2 \arcsin \frac{1}{|z|}
$$

bedeutend verschärft werden kann.

Man hat nämlich zunächst, wie an der angeführten Stelle,

$$
f^{\prime}(z)=\Pi\left(1-\frac{z_{k}}{z}\right)^{t_{k}},
$$

sodann aber wegen (3)

$$
l\left(f^{\prime}(z)\right)=-\sum_{k} \alpha_{k}\left(\frac{1}{2}\left(\frac{z_{k}}{z}\right)^{2}+\frac{1}{3}\left(\frac{z_{k}}{z}\right)^{3}+\ldots\right)
$$

und durch Zerlegung in den reellen und imaginären Teil

(6) $\quad \operatorname{Arg} f^{\prime}(z)=-\sum_{k} \alpha_{k}\left(\frac{1}{2} \cdot \frac{\sin 2\left(\varphi_{k}-\varphi\right)}{|z|^{2}}+\frac{\sin 3\left(\varphi_{k}-\varphi\right)}{|z|^{3}}+\ldots\right)$

$$
\left|\operatorname{Arg} f^{\prime}(z)\right| \leqslant 2\left(l\left(\frac{|z|}{|z|-1}\right)-\frac{1}{|z|}\right)
$$

Diese letztere Schranke ist zwar für $|z|$ nahe eins viel größer als die von Herrn Birnbaum gefundene (3), wird jedoch mit größerem $|z|$ kleiner und nimmt wie $|z|^{-2}$ ab, wenn $|z|$ ins Unendliche geht.

Sie wird übrigens sehr bald kleiner, nämlich bereits sicher für $|z| \geqslant 1 \cdot 188538$. Die Differenz der Schranken (7) weniger (4) beträgt nämlich bei $|z|=1 \cdot 1885537$ nur $14 \cdot 10^{-7}$, für $|z|=1 \cdot 188538$ jedoch $-72.10^{-7}$, woraus sich der etwas schärfere Wert $1 \cdot 1885372$ ergiebt, für welchen beide Schranken sehr nahe einander gleich werden, aber (7) bereits kleiner wird.

* Die oben angegebene Schranke lässt sich ohne große Mühe bedeutend verschärfen.

Aus

$$
l f^{\prime}(z)=\Sigma \alpha_{k}\left(l\left(1-\frac{z_{k}}{z}\right)+\frac{z_{k}}{z}\right)
$$

ergiebt sich nämlich, wenn $z_{k}=e^{i \varphi_{k}}, z=r e^{i \varphi}$ gesetzt wird,

(9) $\quad \operatorname{Arg} f^{\prime}(z)=-\sum \alpha_{k}\left(\operatorname{arctg} \frac{\sin \left(\varphi_{k}-\varphi\right)}{r-\cos \left(\varphi_{k}-\varphi\right)}-\frac{\sin \left(\varphi_{k}-\varphi\right)}{r}\right)$, wobei der Hauptwert des arctg zu nehmen ist.

Es ist also das Maximum des Absolutwertes von

$$
\Phi=\operatorname{arctg} \frac{\sin \varphi}{r-\cos \varphi}-\frac{\sin \varphi}{r} \quad(0 \leqslant \varphi \leqslant \pi)
$$

zu suchen. 
Man findet dafür die Gleichung

$$
2 r \cos ^{2} \varphi-\cos \varphi-r=0,
$$

welche man auch schreiben kann

$$
r \cos 2 \varphi=\cos \varphi
$$

Ihre beiden Lösungen sind

$$
\cos \psi_{1}=\frac{1}{4 r}+\sqrt{\frac{1}{2}+\frac{1}{16 r^{2}}}, \cos \psi_{2}=\frac{1}{4 r}-\sqrt{\frac{1}{2}+\frac{1}{16 r^{2}}}
$$

und es ist

$$
\cos \psi_{1} \cos \psi_{2}=-\frac{1}{2}, \quad \cos \psi_{1}+\cos \psi_{2} \cdots \frac{1}{2 r} .
$$

Die Winkel $\psi_{1}$ und $\psi_{2}$ wachsen beide gleichzeitig mit $r$ und es ist $0 \leqslant \psi_{1} \leqslant \frac{\pi}{4}, \frac{2 \pi}{3} \leqslant \psi_{2}<\frac{3 \pi}{4}$. Hieraus findet sich nach einiger Umformung, wenn die $\psi_{1}$ und $\psi_{2}$ entsprechenden Werte von $\Phi$ mit $\Psi_{1}$ und $\Psi_{2}$ bezeichnet werden,

$$
\begin{gathered}
\Psi_{1}=\frac{\pi}{2}-2 \psi_{1}-\frac{\sin \psi_{1}}{r}, \quad \Psi_{2}=\frac{3 \pi}{2}-\psi_{2}-\frac{\sin \psi_{2}}{r} \\
\Psi_{1}+\Psi_{2}=2 \pi-2\left(\psi_{1}+\psi_{2}\right)-\sin \left(2 \pi-2\left(\psi_{1}+\psi_{2}\right)\right)>0
\end{gathered}
$$

Ferner ist

$$
\frac{d \Psi_{1}}{d \psi_{1}}=\frac{1-4 \cos ^{4} \psi_{1}}{\cos ^{2} \psi_{1}}, \frac{d \Psi_{2}}{d \psi_{2}}=\frac{1-4 \cos ^{4} \psi_{2}}{\cos ^{2} \psi_{2}},
$$

so daß mit Rücksicht auf die Intervalle von $\psi_{1}$ und $\psi_{2}$ der erste Wert beständig zunimmt, der zweite beständig abnimmt.

Da nun $\Psi_{1}$ und $\Psi_{2}$ für $r=1$ entgegengesetzte Zeichen haben und für unendliches $r$ beide verschwinden, so ist $\Psi_{1}$ immer positiv, $\Psi_{2}$ immer negativ und wegen der letzten Formel in (13) immer $\Psi s_{1} \geqslant\left|\Psi_{2}\right|$.

Daher kommt nur $\Psi_{1}$ in Betracht und man erhält als Schranke

$$
\left|\operatorname{Arg} f^{\prime}(z)\right| \leqslant 2\left(\frac{\pi}{2}-\psi_{1}-\frac{\sin \psi_{1}}{r}\right)
$$

Ferner ist bei Entwicklung nach fallenden Potenzen von $r$

$$
w_{1}=\frac{1}{2 r^{2}}+\ldots
$$

Die oben erhalten Schranke ist wohl die schärfste, welche auf diesem Wege zu erhalten ist.

Man erhält jedoch eine bequemere und für das ganze Intervall von $r$ gültige auf folgendem Wege:

Es ist

$$
r^{2} \Psi_{1}=\frac{\cos ^{2} \psi_{1}}{\cos ^{2} 2 \psi_{1}}\left(\frac{\pi}{2}-2 \psi_{1}-\operatorname{tg} \psi_{1} \cos 2 \psi_{1}\right)
$$

Setzt man hier $\frac{\pi}{2}-2 \psi_{1}=(\omega)$, so erhält man

$$
r^{2} w_{1}=\frac{1}{2}\left(\frac{1}{\sin ^{2} \omega}+\frac{1}{\sin \omega}\right) \omega-\frac{1}{2} \operatorname{ctg} \omega
$$

Entwickelt man hier nach steigenden Potenzen von $\omega$, was wegen $0 \leqslant(1) \leqslant \frac{\pi}{2}$ gestattet ist, so fällt $\omega^{-1}$ weg, sämmtliche Koeffizienten werden positiv und es kommt

$$
r^{2} \Psi_{1}=\frac{1}{2}+\frac{1}{3} \omega+\frac{1}{12} \omega^{2}+\ldots
$$

Hieraus entnimmt man, daß $r^{2} \Psi_{1}$ mit $\omega$ wächst und daher seinen größten Wert, nämlich $\frac{\pi}{2}$, für $\Psi=0$ das ist $\omega=\frac{\pi}{2}$ erreicht.

Somit ist $\Psi_{1}<\frac{\pi}{2} r^{-2}$ und

$$
\left|\operatorname{Arg} f^{\prime}(t)\right| \leqslant \frac{\pi}{r^{2}}
$$

Diese letztere Schranke wird der unter (7) gegebenen bei $r=1 \cdot 14341$ nahezu gleich, um sie dann zu überschreiten; vorher ist sie natürlich kleiner. *

(Reçu par la Rédaction le 17. 12. 1931; le passage entre astérisques * * a été ajouté le 10. 6. 1933). 